Article

\title{
Adaptable Underwater Networks: The Relation between Autonomy and Communications
}

\author{
Alexander Hamilton ${ }^{1, *}$, Sam Holdcroft ${ }^{1}$, Davide Fenucci ${ }^{2}{ }^{(1)}$, Paul Mitchell ${ }^{3}$, Nils Morozs ${ }^{3}(\mathbb{D}$, \\ Andrea Munafò ${ }^{2,4}$ and Jeremy Sitbon ${ }^{5}$ (D) \\ 1 Defence Science and Technology Laboratory, Portsdown West, Portsdown Hill Road, \\ Fareham PO17 6AD, UK; sjholdcroft@dstl.gov.uk \\ 2 National Oceanography Centre, European Way, Southampton SO14 3ZH, UK; davfen@noc.ac.uk (D.F.); \\ andrea.munafo@seebyte.com (A.M.) \\ 3 Department of Electronic Engineering, University of York, York YO10 5DD, UK; \\ paul.mitchell@york.ac.uk (P.M.); nils.morozs@york.ac.uk (N.M.) \\ 4 Seebyte Ltd., Orchard Brae House, Edinburgh EH4 2HS, UK \\ 5 Planet Ocean Ltd., 16, Camberley Business Centre, Camberley GU15 3DP, UK; jeremy@planet-ocean.co.uk \\ * Correspondence: ajhamilton@dstl.gov.uk; Tel.: +44-(0)1980-951168
}

Received: 4 September 2020; Accepted: 2 October 2020; Published: 10 October 2020

check for updates

\begin{abstract}
This paper discusses requirements for autonomy and communications in maritime environments through two use cases which are sourced from military scenarios: Mine Counter Measures (MCM) and Anti-Submarine Warfare (ASW). To address these requirements, this work proposes a service-oriented architecture that breaks the typical boundaries between the autonomy and the communications stacks. An initial version of the architecture has been implemented and its deployment during a field trial done in January 2019 is reported. The paper discusses the achieved results in terms of system flexibility and ability to address the MCM and ASW requirements.
\end{abstract}

Keywords: underwater communication; acoustic communication; channel sensing; adaptive communications; autonomous underwater vehicles; underwater networks; interoperability

\section{Introduction}

Technology in the underwater domain is rapidly increasing in capability, with ever developing robotics technology enabling a wider variety of actors to access the subsurface domain. This has facilitated a significant increase in capability that can be provided for underwater missions. One of the key challenges to overcome given the increased appetite for underwater missions supported by robotic systems is underwater communications. It has long been recognised that any form of communications is difficult underwater [1,2] and there is a severe trade off between range and achievable data rates, primarily due to the transmission medium and its variability with time and location $[3,4]$. Acoustic communication is the most common medium choice, and is the only method for communicating over more than a couple of hundred metres. Most underwater nodes (whether they be static or mobile) will be outfitted with an acoustic modem, with some also receiving an RF or optical modem too. Recent efforts have led to the deployment of networks of these nodes, usually networks of seabed sensors communicating data to a surface station.

Underwater Acoustic communication (UWA) is typically limited in range and coverage by the ocean conditions in which they exist. For this reason, while they are often utilised to support Autonomous Underwater Vehicles (AUVs) operations, they have been traditionally seen, from an autonomy perspective, as an additional mission constraint, often limiting the achievable objectives.

Within UWA channels, one of the biggest considerations for whether communications can be achieved are the environmental parameters, such as bathymetry or whether an acoustic line of 
sight exists between two nodes. The use of autonomous vehicles presents an opportunity to adapt the physical network topology to these environmental parameters. Unlike traditional networking infrastructure, AUVs are mobile platforms and could be tasked to position themselves to support a communications infrastructure.

Much prior work exists on how these underwater communications networks can be used to support autonomous behaviour [5,6], but this paper presents a different viewpoint: How can the communications networks and the autonomous behaviours interact together to maximise the mission effectiveness? While most of the previous literature has focused on using communications to enable cooperation of AUVs, this work aimed at proposing a tighter relation between autonomy and communication, so that, for example, autonomous behaviours can also be used to support communications. This is achieved through a service-oriented architecture that integrates together, through a well-defined set of services, the communications layers (physical and network) with the autonomy.

The context and motivation behind this work are represented by two uses cases sourced from military applications, but that are representative of a broader set of scenarios: Mine Counter Measurements and Anti-Submarine Warfare.

\subsection{Related Work}

From an autonomy perspective, communication requirements have been integrated in the vehicle's on-board control system following two different conceptual methods resulting in a different capability for the autonomy to balance the mission requirements with their communication needs.

\subsubsection{Autonomy and Communications as Separate Entities}

In the typical approach, the communication and autonomy layers are separated. The autonomy system uses communications mainly to share data with other vehicles or nodes of the network and/or with the $\mathrm{C} 2$ centre. The autonomous decision-making process is rarely communication aware, which means that mission-level decisions or the vehicle trajectory planning are only based on the overall mission goal. When communications are included in the autonomy framework, they are often seen as an additional planning or bandwidth constraint. Vehicles might be requested to stay within a predefined communication range $[7,8]$, or, as in the case of gliders, to periodically surface to get radio or satellite communications, or their trajectory is planned so that they can pass close to gateways and/or communications relays to offload data or update their mission parameters. For example, authors in [9] propose to include communication constraints through Rendezvous Points (RPs) to address some of the communications limitations of Mine Counter Measure (MCM) scenarios. In this case, the vehicles dynamically agree on a meeting point to exchange target locations, to decide how to allocate remaining and new tasks and to agree on the next meeting point and location. The disadvantage of this method is that vehicles are only guaranteed to be in communication at the RPs. This limits the in-mission collaboration and loses resources as the vehicles have to travel to the RPs.

Communications as a sharing bottleneck for autonomous robots is tackled, among others, in [10] where local roadmaps are incrementally improved by passing messages among the network nodes. This makes it possible for each node to calculate a feasible path across the area of interest in a distributed way. A similar approach is also followed in the Neptune autonomy framework [11], one of the main commercially available autonomy frameworks. To limit communications, each robotic node opportunistically updates its internal representation of the world whenever new information becomes available. A summary of this internal representation is then shared with everyone else. The system keeps on transmitting the same information until the shared summary converges to a unique solution.

A different approach to overcome the bandwidth limitations and large time delays of underwater communications is used in case robots can physically interact each others. Among others, authors in [12] describe how to achieve multi-agent robotic coordination relying only on implicit communication that is possible through the physical interaction of the robots with a commonly grasped object. A similar 
problem is also tackled in [13], where a cooperative control policy for Unmanned Underwater Vehicles is devised to make explicit usage of the limited communication bandwidth available.

When, vice versa, the problem is approached from a communication perspective, autonomous vehicles are seen as mobile nodes of the network that are able to relay information. In the context of submarine surveillance and monitoring [14], where a prompt communication of critical information is paramount, AUVs are used to retrieve high-value information with specific time deadlines after which the data becomes no longer relevant. An Integer Linear Programming method is used to calculate a path for the AUV so that the Value of Information (VoI) of the data delivered to the sink is maximised, while considering realistic data communication rates, distances and surfacing constraints.

An interesting approach is the one proposed in [15] with the design of a Cognitive Communications Architecture (CCA) that can support deployment of advanced autonomous underwater solutions making use of smart, adaptive and secure underwater networking strategies. While the communication architecture is designed to be modular and able to provide information at different levels, including the autonomy, the authors do not explicitly consider a link with the autonomy system.

To try and tackle some of these challenges, [16] presented a unified C2 architecture that uses a compressed short message encoding scheme (Dynamic Compact Control Language or DCCL) to transfer commands and receive vehicle status, and easily adaptable to heterogeneous vehicles. The objective is to inform the operators so that better decisions can be taken at the $\mathrm{C} 2$ level. A different approach is the one proposed in [17], where an implicit data-compression and transmission protocol is proposed to carry out environmental monitoring missions such as adaptive sampling of physical and chemical parameters in the water. Communications between the vehicle and the control centre are performed in a semi-autonomous mode: as long as the measured data matches the predicted data, the robot remains silent and only sends packets to confirm that it has arrived at navigational check-points. In the case that any unpredicted event happens, the robot sends a priority packet containing the new environmental/kinematic data. The control centre receives the updated information and decides whether to update the vehicle's mission or not. A communication framework that looks for favourable network topologies before starting to communicate is proposed in [18]. Results exploit the predictability of the AUV trajectories to delay packet transmissions to geometrical positions that would improve communication performance (e.g., shorter distance). Active network geometry optimisation is proposed in [19]. In this case, a subset of the nodes can relocate to dynamically adapt their locations to maximise localisation and communication performance. A planning algorithm that adapts the network geometry and optimises the transmission times is proposed in [20].

In most of these scenarios however, the communication stack is effectively unaware of the overall mission goal, and only concerned about the efficiency of the transmission and/or reception, possibly optimising the vehicle localisation for message routing or optimising gateway locations. This is, for example, proposed in [21] with a mobile bridging node within a network of fixed heterogeneous acoustic modems.

\subsubsection{Autonomy and Communications in Synergy}

A different approach considers the autonomy and the communication systems as mutually interacting entities rather than being independent. As operations with multiple AUVs become more common, a lot of effort is directed towards finding solutions to increase the mission efficiency in terms of time and quality of the data collected. In this regard, sharing information across autonomy and communication layers can be very beneficial. First of all, providing the decision-making process with a holistic view of the mission evolution allows a broader definition of the mission objectives, including parameters such as desired communication performance, minimisation of the navigational drift, etc. Furthermore, a mission-/task-aware communication system can improve the channel usage to optimise the throughput of the network, e.g., adjusting the routing paths to include vehicles within communication range only. Moving in this direction, authors in [22] propose to run an acoustic model 
in real-time to provide estimates of the acoustic environment under changing environmental and situational scenarios. While the output of the model is combined together with other mission objectives to maintain point-to-point acoustic connectivity throughout the mission, there is not explicit aim to tackle the problem at network level, when more than two nodes are present. Another example of interaction between autonomy and communications is the one proposed in [6]. In this case, the acoustic network is able to provide additional, autonomy oriented, services, namely localisation and navigation. The localisation capability is added on top of an existing network, without imposing constraints on its structure and operation, and to support AUV navigation without constraining the network design and with a minimum communication overhead.

\subsection{Main Contributions}

This paper aimed at discussing some of the main requirements for communications and autonomy in maritime environments. These requirements come from two use cases that have been sourced from military requirements for dynamic underwater networks, namely Mine Counter Measures (MCM) and Anti-Submarine Warfare (ASW). We use these use cases to provide motivation and context for this work, but they are representative of a much wider variety of uses. To address these requirements, this paper proposes to couple autonomy and communications through a service-oriented architecture that aims at explicitly integrating together autonomous behaviours and communication protocols. The system implementation with an initial set of services and its deployment on a network of AUVs is discussed and preliminary results are reported.

\subsection{Organisation of the Paper}

Section 2 describes the use cases that have been the guiding motivation for the rest of the paper. Section 3 describes research on fundamental acoustic communications-propagation through an underwater acoustic channel and medium access control. This section also introduces the concept of a data mule, as well as exploring the trade offs between traditional communications and capability offered by an autonomous data mule. Section 4 explores how autonomous systems can be tasked to enhance communications. Section 5 provides a case study on recent trials where the described system has been deployed on a network of AUVs. Section 6 discusses the results and highlights shortcomings and additional challenges that have not been fully investigated in this work. Finally a conclusion is given, containing information about ongoing research programmes.

\section{Use Cases}

Two use cases are presented in this paper, Mine Counter Measures and Anti-Submarine Warfare. A traditional Mine Counter Measure (MCM) operation involves a fleet of surface and subsurface platforms working in unison to identify and neutralise subsurface targets. This will most often be in completely unknown environments, with little opportunity for reconnaissance. Anti-Submarine Warfare (ASW) operations can take place in both known and unknown environments. There may be historical bathymetry data available, but this is not guaranteed. This operation relies of a fleet of subsurface vehicles collecting data on possible targets, and reporting this to a remote data sink. There may be additional requirements for covertness. Both of these operations are expected to be carried out in littoral waters, and any live data will need to be distributed across the network.

\subsection{Autonomous Mine Counter Measures (MCM)}

In this scenario (Figure 1), a fleet of Autonomous Underwater Vehicles (AUVs) are deployed as a networked swarm into the operating environment. The mission is controlled from a surface vessel, which maintains contact with the underwater fleet via acoustic communications. The AUV network must gather data on the targets; this data may consist of side-scan sonar data, images from on board cameras and location of the target from on board position estimates. The data can either be processed locally or transmitted to the surface vessel for further analysis. The geographical environments for 
which MCM is likely to be utilised in will be shallow water environments, where an acoustic line of sight may not exist. Furthermore, to reserve battery power on AUVs, they will most likely wish to remain submerged and on task, being provided with Command and Control (C2) and navigation information sub-surface. All nodes should be aware of the capabilities possessed within a mixed fleet autonomous MCM environment and how each can be tasked to most efficiently achieve the overall mission aim.

Local processing would involve running some target recognition algorithms over the data, which would significantly reduce the amount of data needed for transmission back to the base station. However, this incurs significant processing overhead and may be infeasible on low powered platforms, and the operators must accept the risks posed by inaccurate target recognition algorithms. Transmitting all of the data back to the surface vessel places a significant burden on the low data rate acoustic communications links available.

It is envisaged that for future autonomous MCM operations, a mixed fleet of AUVs will be used with more than 10 platforms, for accurate and rapid assessment of the environment.

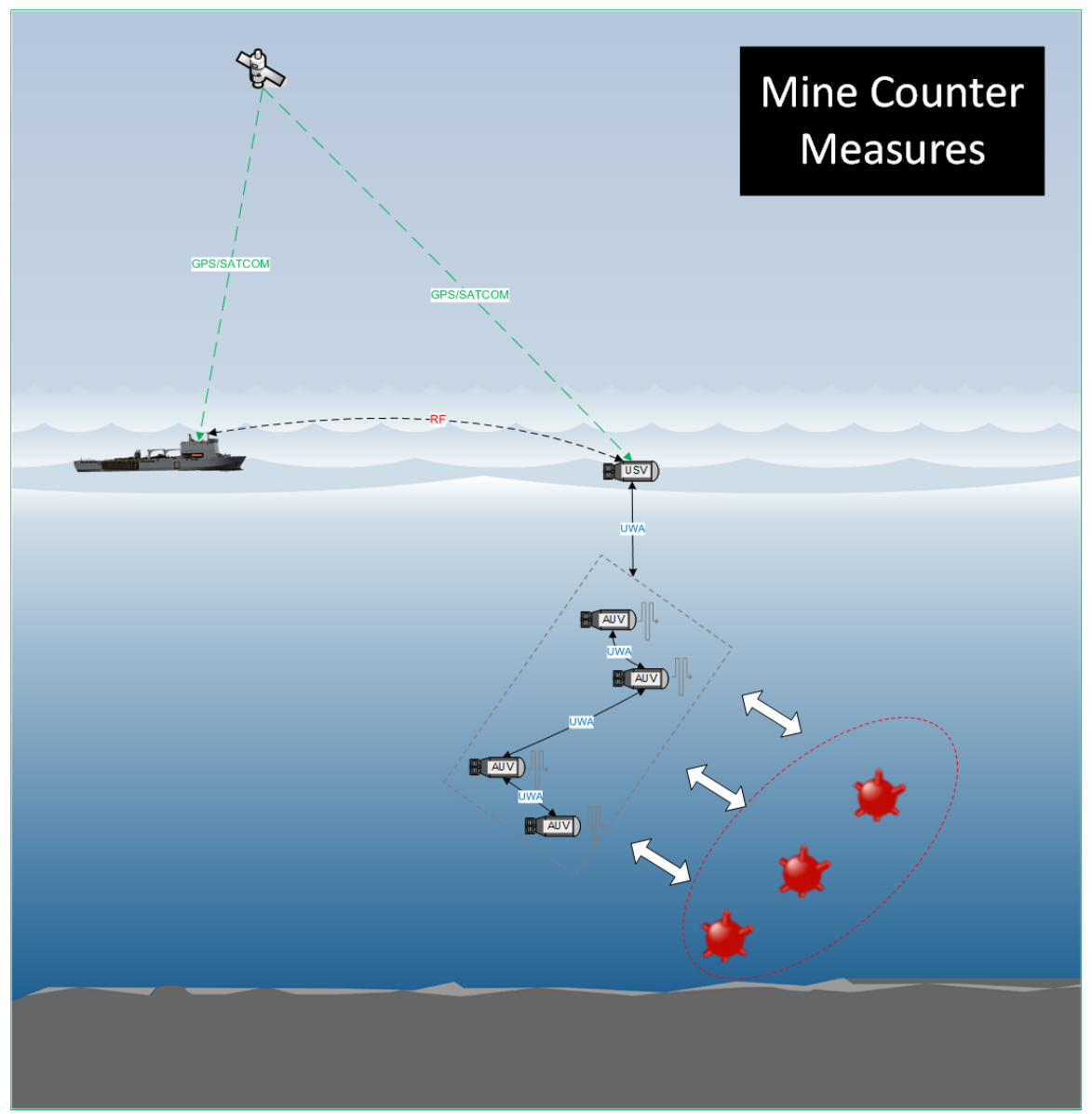

Figure 1. Mine Counter Measure use case. The Autonomous Underwater Vehicles (AUVs) communicate over acoustic channels, reporting collected data back to the command station through an Unmanned Surface Vehicle (USV). This surface vehicle is localised via GPS.

\subsection{Anti-Submarine Warfare (ASW)}

The ASW scenario (Figure 2) comprises a network of AUVs stationed in a geo-stationary defined area, trying to detect any submarine platforms that are present. This detection could be achieved through passive arrays, as part of a multi-static active network, or other variations. This data must then be returned to an operational commander to provide Situational Awareness (SA). An ASW operation could persist over a long time period, and might not have a defined end date. The AUV nodes will 
likely be collecting sonar data or passive hydrophone recordings. Once again, this data can either be processed locally or transmitted to the command node for processing.

Unlike the MCM scenario, actual detection events are likely to be very rare. Instead, most traffic will likely be network management (vehicle status, location reporting, etc.) or false positives. The network will either need to provide a data rate high enough to support the transmission of these false positives, or to filter them out. Additionally, there may be a requirement for the AUV network to operate with a reduced acoustic signature to reduce the likelihood of an adversary detecting the presence of the ASW operation.

As with MCM, an ASW scenario utilising autonomous systems will probably comprise a large mixed fleet in excess of 10 platforms. Furthermore, ASW operations are likely to occur in slightly deeper water whereby shadow zones caused by acoustic sound channels will come into effect, both disrupting communications, but also being able to hide potential contacts.

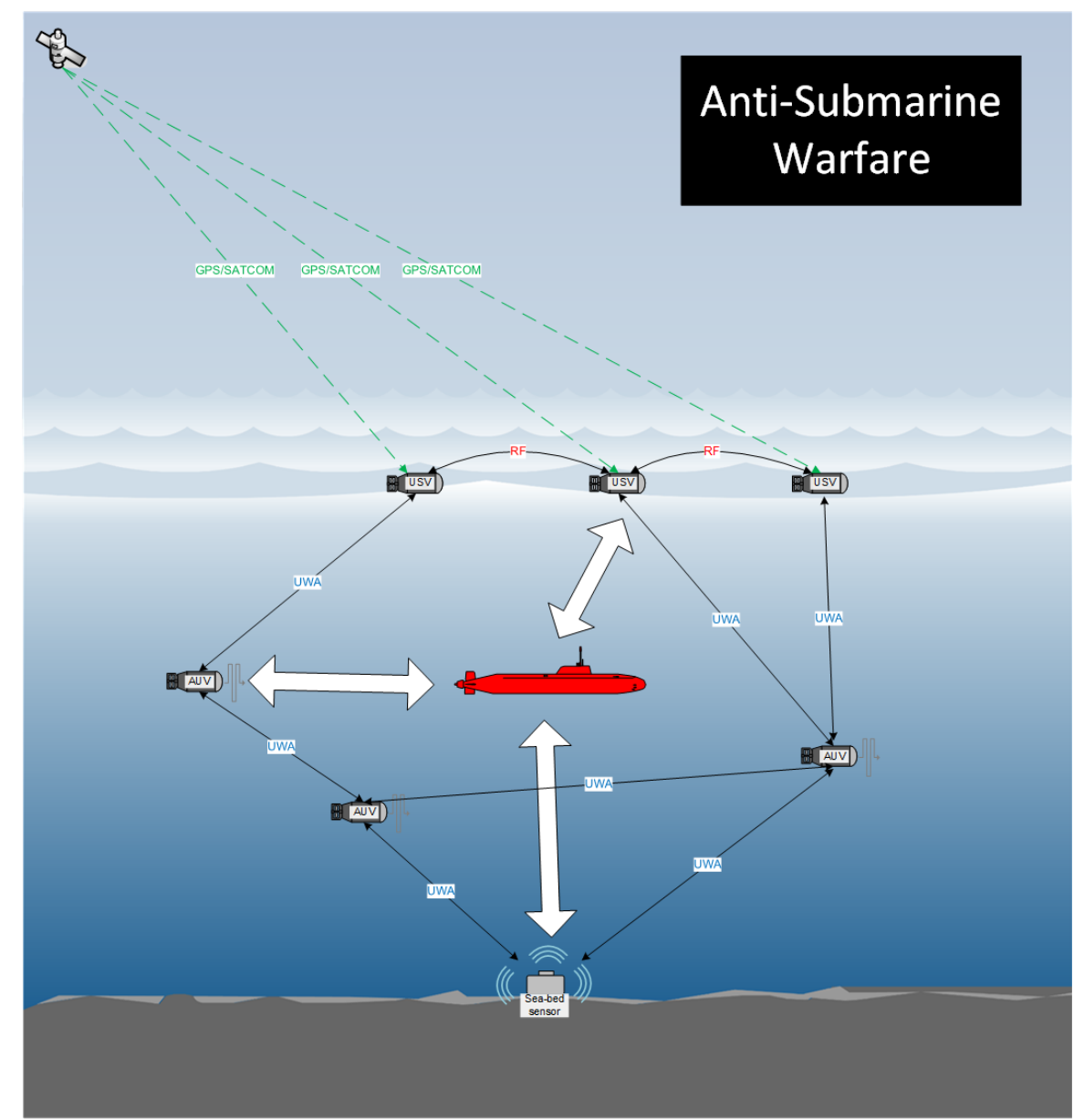

Figure 2. Anti-Submarine Warfare use case. A network of USVs identifies a hostile platform, and informs a remote command post via satellite link.

\section{Underwater Communication and Network Adaptation}

The dominant physical property limiting the communication and networking capabilities of underwater systems is the slow propagation of acoustic waves. In contrast with terrestrial radio networks with a propagation speed of $3 \times 10^{8} \mathrm{~m} / \mathrm{s}$, acoustic waves propagate through water at approximately $1500 \mathrm{~m} / \mathrm{s}$, i.e., slower by a factor of $2 \times 10^{5}$. This presents a significant challenge in the design of UWA communication networks.

In addition to the physical layer challenges, such as frequency selective fading, long multipath delay spread and the Doppler effect [23-25], the slow propagation of acoustic waves presents a 
significant challenge in network protocol design, i.e., coordinating UWA communication among multiple acoustic communication nodes potentially spaced kilometres apart. These challenges, in turn, have a direct impact on the design of multi-vehicle coordination algorithms that rely on information exchange.

The rest of the section introduces the main challenges of underwater communications from a physical propagation standpoint and from the medium access control perspective. These play a key role in determining suitable strategies at the autonomy level and lay out the foundations of the system described in Section 4.

\subsection{Underwater Acoustic Propagation}

In addition to being slow, the propagation speed of acoustic waves depends on the temperature, pressure and salinity of the water and is, therefore, variable in space and time [26]. Figure 3 shows an example of two sound speed profiles (SSPs) derived by Dushaw [27] from the 2009 World Ocean Atlas data for January and July, both in the North Sea at $\left(55.5^{\circ} \mathrm{N}, 2.5^{\circ} \mathrm{E}\right)$. In this example (relatively shallow water), the water temperature is a dominant parameter affecting the sound speed, introducing considerable variability in the SSP between January and July. Since the water near the sea surface is warmer in July, there is a distinct negative sound speed gradient caused by this thermocline. Such variations in the sound speed at different depths cause refraction of acoustic waves towards the lower sound speed region. This, in turn, results in curved propagation trajectories that may sometimes form waveguides or deep sounds channels around a particular depth (where the sound speed is at its minimum). For example, the SSPs shown in Figure $3 \mathrm{~b}$ cause downwards refraction towards the seabed in July but a slight upwards refraction in January, which can have a considerable impact on the network connectivity and the link quality.

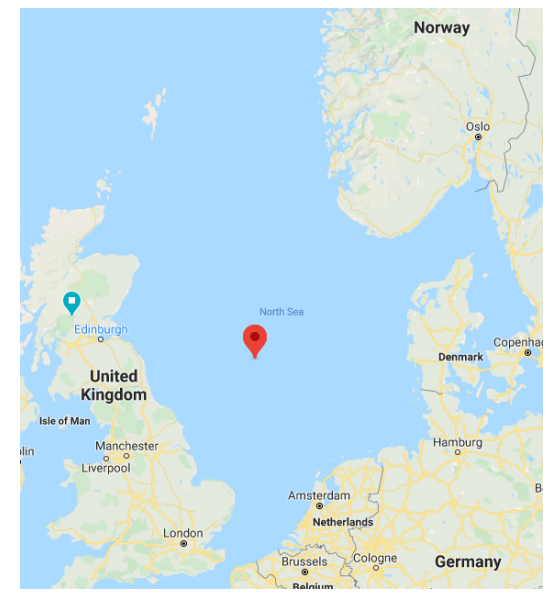

(a) Google Maps location

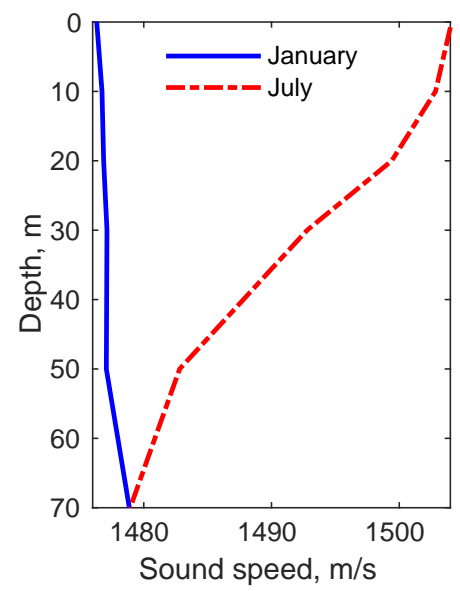

(b) Sound speed profile

Figure 3. Sound speed profile (SSP) examples in January and July, based on the temperature, pressure and salinity data in the North Sea at $\left(55.5^{\circ} \mathrm{N}, 2.5^{\circ} \mathrm{E}\right)$.

Figure 4 shows how the spatial distribution of the UWA channel quality may vary, depending on the SSP and the location of the source in the water column. The contour plots show the Signal-to-Noise Ratio (SNR) computed using the channel modelling framework described in [28] based on BELLHOP beam tracing [29] with randomly generated surface waves and the January and July SSPs from Figure $3 \mathrm{~b}$. The outputs of BELLHOP were post-processed using a $24 \mathrm{kHz}$ centre frequency and $7.2 \mathrm{kHz}$ bandwidth, i.e., representative of typical wideband UWA transmissions [30], by integrating the absorption loss for every traced multipath component across the whole frequency band to yield the wideband received signal power (see [28]). The SNR values were computed by dividing the wideband received signal power by the noise power calculated using the ambient UWA noise model 
in [31], assuming $10 \mathrm{~m} / \mathrm{s}$ wind speed, 0.5 shipping activity factor, $24 \mathrm{kHz}$ centre frequency and $7.2 \mathrm{kHz}$ bandwidth.

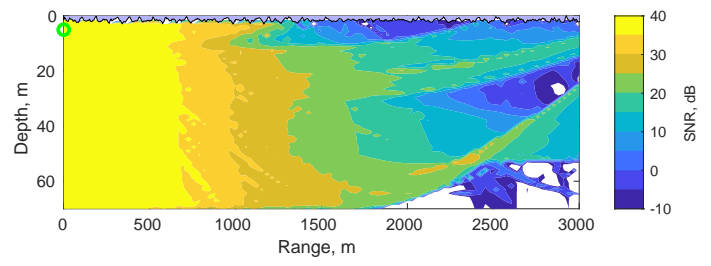

(a) January SSP, source at $5 \mathrm{~m}$ depth

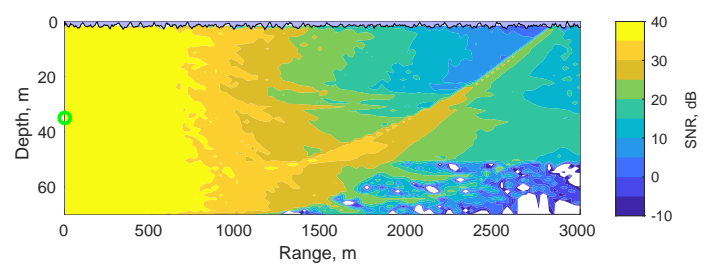

(c) January SSP, source at $35 \mathrm{~m}$ depth

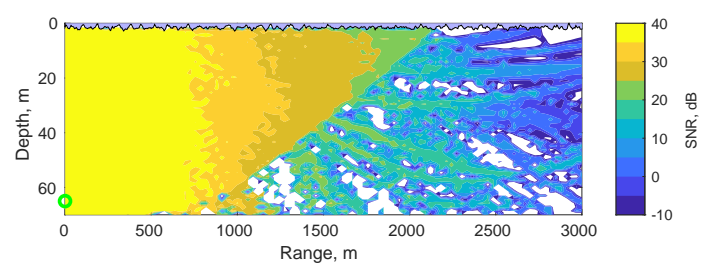

(e) January SSP, source at $65 \mathrm{~m}$ depth

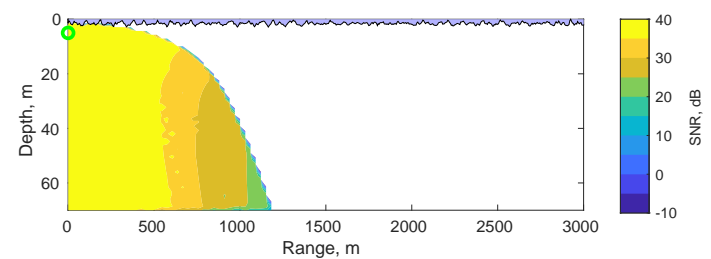

(b) July SSP, source at $5 \mathrm{~m}$ depth

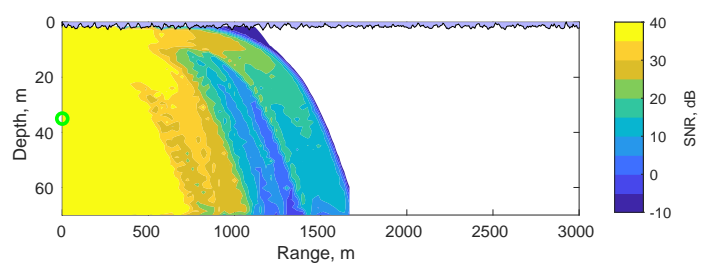

(d) July SSP, source at $35 \mathrm{~m}$ depth

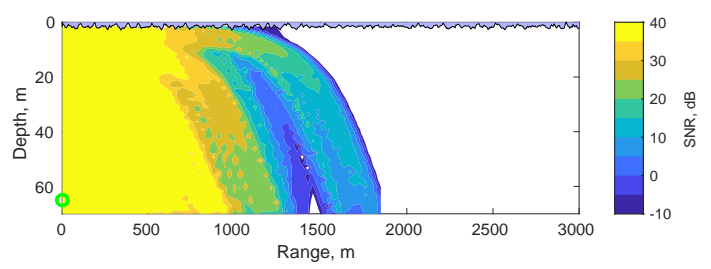

(f) July SSP, source at $65 \mathrm{~m}$ depth

Figure 4. Signal-to-Noise Ratio (SNR) computed using beam tracing simulations of the North Sea environment. The communication coverage and channel quality are significantly affected by the refraction of acoustic waves and the node positions in the water column. Signal parameters: $180 \mathrm{~dB}$ re $1 \mu \mathrm{Pa} @ 1 \mathrm{~m}$ source level, $24 \mathrm{kHz}$ centre frequency, $7.2 \mathrm{kHz}$ bandwidth.

Firstly, the plots in the right-hand column of Figure 4 demonstrate the effect of the negative sound speed gradient in July, with the downwards refraction limiting the communication range to approximately 1-1.8 $\mathrm{km}$ depending on the source and receiver depths. Note, these simulations used the generic BELLHOP seabed setting, a flat surface modelled as an acousto-elastic half-space with $1600 \mathrm{~m} / \mathrm{s}$ sound speed and $1 \mathrm{~g} / \mathrm{cm}^{3}$ density (representative of sand-silt [32]). A different bathymetry may result in usable UWA communication links via reflections off the seabed [33,34]. In contrast with the July results, Figure $4 \mathrm{a}, \mathrm{c}$,e show that the lack of thermocline in the January SSP results in more horizontal propagation of the acoustic waves and, therefore, in most cases a longer communication range (with the exception of seabed to seabed communication, where the downwards refraction in July is generally more favourable).

Secondly, the different rows of plots in Figure 4 show the effect of varying the source depth on the coverage area and the channel quality of UWA communication. The plots demonstrate the formation of sound channels and acoustic shadows at different geographical locations depending on the SSP and the source depth. For example, the slight upwards refraction in January results in sound channels with high SNR at relatively long ranges (e.g., Figure $4 a$,c), which can be exploited for high quality UWA communication if the source and the receiver are located in favourable positions in the water column. Conversely, it would be desirable to position the underwater nodes to avoid the acoustic shadow zones and areas of low SNR to boost the reliability of the communication links. One of the key features of the autonomous subsea systems considered in this paper is their capability to adapt to the given UWA propagation environment and improve the quality of UWA communication among the network nodes with minimum disruption to their primary missions, as discussed in Section 4. 


\subsection{Challenges in Medium Access Control}

Most conventional medium access control (MAC) protocols, developed for terrestrial radio systems, are far less efficient in the UWA environment. For example, channel reservation based protocols waste a significant part of the available channel capacity while nodes wait for control signals to propagate through the slow acoustic medium to establish a communication link, e.g., Request-to-Send (RTS), Clear-to-Send (CTS), acknowledgements, etc. These waiting times result in significant loss of throughput and poor channel utilisation [35-37]. Furthermore, Carrier Sense Multiple Access (CSMA) techniques, where nodes "listen" for the presence of other packets on the shared channel before transmitting, are also inefficient in Underwater Acoustic Networks (UANs). In order for UAN nodes to accurately detect when the channel is free using carrier sensing, excessive guard intervals are required to compensate for the long propagation delays [35]. A different class of MAC protocols, more suitable for high throughput scenarios, is based on the principle of Time Division Multiple Access (TDMA), where the nodes are scheduled to transmit their data packets in particular time slots such that the packets arrive at the intended receivers without collisions [38-40]. Schedule-based MAC schemes do not involve ad hoc contention for communication resources, thus removing the need for control packets in order to establish collision-free links. However, the main drawback of typical TDMA protocols is the need for extensive guard intervals to account for long propagation delays of acoustic waves, which often has a large negative impact on the network throughput. They also require clock synchronization at all network nodes, which is more challenging in UASNs than terrestrial systems $[35,41]$.

Other approaches to facilitating collision-free (orthogonal) medium access are Frequency Division Multiple Access (FDMA) [42], where multiple nodes are allocated different portions of the frequency spectrum, and Code Division Multiple Access (CDMA) [43], where multiple nodes transmit/receive simultaneously in the same spectrum, but encoding their signals with spreading sequences to enable signal separation at the receiver. The use of FDMA in UWA networks is severely limited by another fundamental physical property of UWA communication: the low frequency bandwidth (typically of the order of several kHz) $[35,42]$. Consequently, the typical FDMA capability in UWA communication systems is limited to a small number of frequency channels that is likely to be insufficient for fully orthogonal FDMA operation of UWA networks. The classical CDMA approach, based on Direct Sequence Spread Spectrum (DSSS), is also significantly more challenging in the UWA domain compared with terrestrial systems. Large, time-variant differences in the propagation delays among multiple nodes and the limitation in the temporal channel coherence make it more difficult to maintain the orthogonality of the DSSS codes and suppress the interference from other nodes [43,44]. Another spread spectrum approach often used in the UWA domain is based on Frequency-Hopped Frequency Shift Keying (FH-FSK) [44,45], where multiple nodes are assigned different frequency hopping patterns in a way that minimizes the probability of collision between two or more nodes. In addition to its capability to support multiple users, FH-FSK is also robust to multipath and impulsive noise, making it a suitable modulation scheme for UWA communications. However, the robustness of FH-FSK comes at the cost of a significant reduction in the raw data rates provided to the underwater nodes.

\subsection{Network Adaptation}

Figure 5 depicts our envisaged adaptability of the subsea networks to the UWA channel conditions, subject to the constraints of other autonomous missions running concurrently on the AUVs. For example, in the MCM use case, the nodes' primary mission is that of collecting data on the targets and to transmit relevant information to the survey vessel as soon as possible. These two, potentially conflicting, objectives have to be balanced out so that the network nodes (the AUVs) would have the capability to alter their course/mission to boost the quality of inter-node communication whenever needed. This can be based on the combination of the following: 
- Internal UWA propagation modelling - the nodes can locally store a set of environmental parameters for the given geographical location, e.g., the SSP and bathymetry, and use them to predict favourable locations/depths for reliable UWA communication, e.g., by performing simulations similar to those in Figure 4 (see also [22] for more details on a real-time implementation for use by AUVs).

- Channel estimation at the receiver-local measurements of the received signal parameters, e.g., signal strength, delay spread, ratio between the direct path and reflected path power, can further enhance the nodes' capability for adapting to their communication environment.

- Channel state feedback — crucially, the channel state measurements can be communicated among the network nodes to provide the capability for closed loop adaptation (the transmitting node adapts based on the channel state information from its intended receiver, and vice-versa). This functionality is challenging to implement in subsea networks due to the high latency of UWA communication which precludes the possibility of near-instantaneous channel state feedback-a standard feature in terrestrial radio networks.

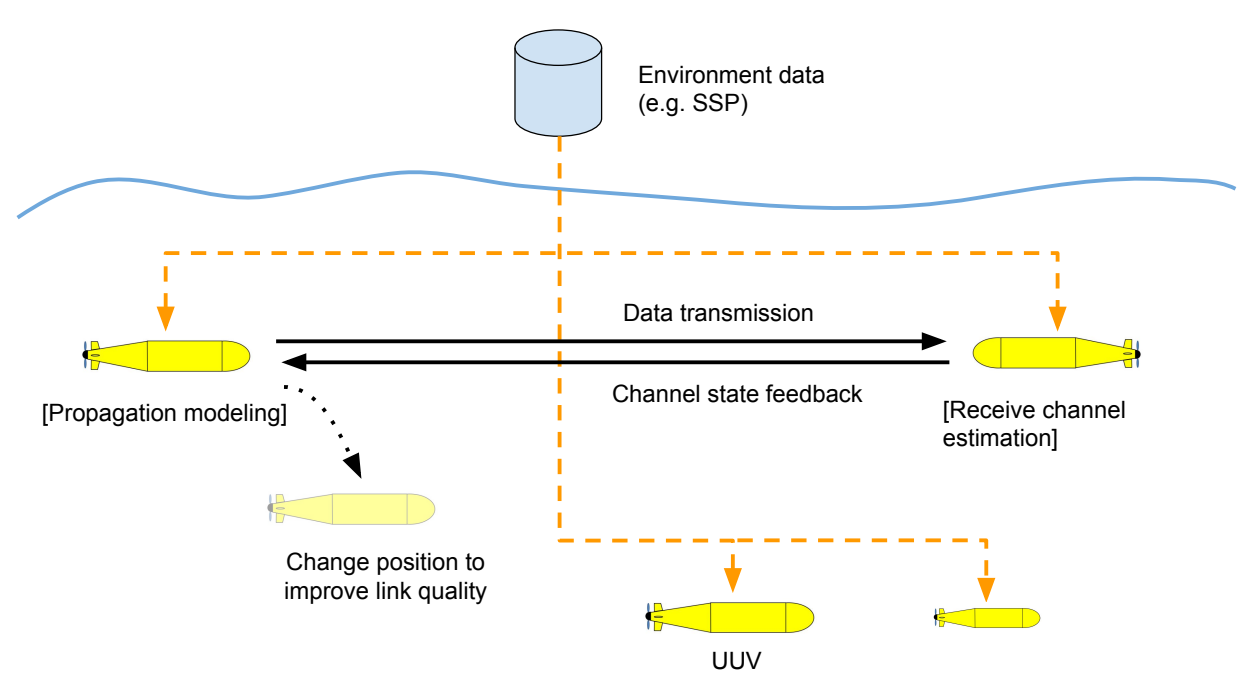

Figure 5. Autonomous node mobility to improve the quality of UWA communication based on: (1) internal propagation modelling, (2) channel estimation, (3) channel state feedback from the receiver.

In addition to improving the reliability of the communication links, the capability of a sub-sea network to adapt to its UWA environment can also be utilised in the MAC and network layers:

- Efficient medium access - the nodes can intelligently adjust their positions to strengthen some communication links, while also "hiding" other, unwanted links. This can reduce the amount of unwanted interference in the network and achieve higher MAC layer throughput and lower end-to-end latency, e.g., via simultaneous scheduling of spatially separated transmissions.

- Adaptive routing - such intelligent node mobility can also be utilised for routing the packets via multiple hops between the source and the destination node with higher end-to-end reliability.

- Reduced non-cooperative detection - the UWA propagation modelling capability of the nodes, based on the knowledge of the environmental parameters, can also be used to decrease the probability of detection of the network by reducing the acoustic energy received outside of the network coverage area, e.g., by moving the nodes higher in the water column in the example in Figure $4 \mathrm{~b}$.

\subsection{Data Mule}

To augment the capability of the proposed UWA systems further, due to limited and range dependent bandwidth of UWA systems, it is useful to understand how data may be carried submerged 
throughout the network as a totality. To this end, it is useful to introduce the concept of a data mule; whereby it is beneficial to have high-latency but (relatively) large amounts of data transfer.

At a simplistic level, a data mule may be considered to be a physical media storage, moving data between two spatially separated network nodes. This type of data mule may also be described as a harvest and deliver data mule as it is harvesting data from one physical area of the network to deliver it to another (Figure 6).

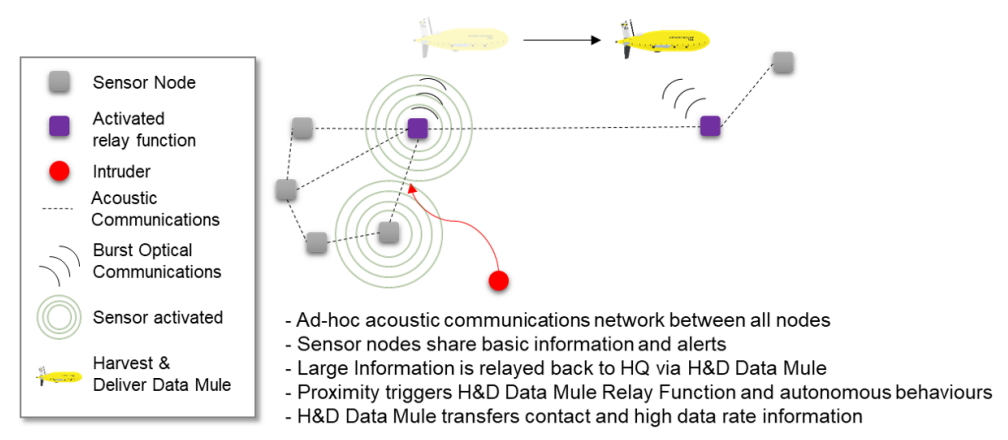

Figure 6. Concept of autonomous harvest and deliver data mule.

Breaking the concept down for underwater networks this creates 5 distinct phases, which are described below:

1. Hailing of the data mule.

2. Initial flight to information source.

3. Information upload.

4. Flight to information sink.

5. Information download from data mule.

The total transmission time is:

$$
T_{\text {total }}=T_{\text {hailing }}+T_{\text {initialflight }}+T_{\text {upload }}+T_{\text {flight }}+T_{\text {download }}
$$

During the hailing phase, the data mule must be hailed to the information source. This must occur with a robust, reliable and reasonably long range, but potentially low data rate underwater communications bearer; as such this task lends itself to UWA communications. During this stage of hailing, the authentication of the information source by the data mule must be a top priority, in order to ensure that the data mule travels to a trusted node; and, appropriate security measures must be applied [46].

Once the hailing stage has completed, and the data mule has been correctly identified by the information source, and the data mule has authenticated the information source which requires the data mule, then the data mule must navigate to a position whereby it can utilise a high data rate communications medium to initiate upload to the data mule. During this initial flight, the data mule will come under the command and control structure of the information source and as such must be able to remain in communications and situational awareness with the information source.

Once the data mule has arrived at a physical location whereby a higher data rate communications medium may be able to be used in order to transmit a large amount of data, the information upload can occur. During this stage, a suitable level of link encryption can be utilised to ensure appropriate security goals can be achieved.

An optical communications bearer is the most appropriate choice of communications during this stage, proving data rates (during the upload) potentially in excess of $1 \mathrm{Gbps}$. Several potential options are discussed in [47] and a practical system that can be utilised off the shelf will likely provide somewhere of the order of $10 \mathrm{Mbps}$, such as the Sonardyne BlueComm 200. 
Once the information content has been safely loaded onto the data mule from the information source, then the data mule must travel to the end destination, or information sink. It is imperative that during this flight, the data mule plots appropriate co-ordinates to ensure safe passage, but also remains in some form of communication with the information source, until an appropriate hand-over of command and control can be achieved. This is to ensure that the data mule can identify another node of any issues in flight, as well as provide another node with situational awareness of the location of the data mule.

Once the data mule arrives at the information sink, the information needs to be downloaded off the data mule. An appropriate authentication process will need to occur between the data mule and the AUV to ensure that the information being downloaded is from the true information source; and also to ensure that the information sink is the true recipient and not an interceptor.

The information can then be downloaded; either by physical retrieval of the information content, via wired means, or likely by optical communications if both assets are submerged.

Whilst the data mule concept is attractive for sending large volumes of data with a high latency, it must be remembered that the overall data rate of the holistic system is determined by a number of factors. If we take into account the total transmission time within (1), then it is possible to define the overall data rate as (2).

$$
\text { DataRate }=\text { DataPacketSize } / T_{\text {total }}
$$

This data rate, in practice, could in fact be less than some of the low data rate protocols that are observed in UWA communications; thereby, as part of the overall mission planning, these factors should be considered before employing such a data mule.

The presence of a data mule can play a key role in a number of applications (see Section 2). Depending on the scenario, it can involve a single dedicated vehicle or be composed of multiple assets that take turns to assume the data mule role as the mission evolves. Regardless, the data mule may be set to periodically transit back and forth to a number of pre-determined locations and harvest mission details from other vehicles without the need to go back to the surface; hence, potentially breaking restrictions on electromagnetic transmissions. Moreover, it can enable multi-squad periodic re-synchronisation, enabling a larger group of possibly separated teams to update their local world view.

\section{Tasking the Network using a Service-Oriented Architecture}

Autonomous operations are critically dependent on underwater communications and networks to be effective and to be trusted. They are the key means to keep operators and robots jointly situated, leading to good decision making by both parties. With limited bandwidths and ranges, data transmissions have to be prioritised and relayed by platforms. Transmissions will be interrupted, leading to periods of communication blackout between platforms. As discussed in Section 3.1, the physics of underwater acoustic propagation plays a key role in the determination of the communication channel characteristics. In turn, the physics of acoustic propagation is strongly influenced by the oceanographic conditions, since the sound speed in the water is a function of temperature, salinity and depth, and these quantities vary both in space and in time. The bursty nature of data flow caused by UWA communications creates requirements for vehicles and operators to use prediction and assumption to take decisions and adapt mission plans on the fly, with the potential for revision once transmissions are restored. In typical ASW or MCM autonomous missions, when the vehicles do not communicate for a pre-specified amount of time, there is usually a safety behaviour that is pre-programmed to abort the mission and to return to a safe point where communication can be re-established. In short, without communications for extended periods, operators and autonomous systems face decisions about mission termination. This results in reduced mission effectiveness, higher costs and slower tempo. The lack of integration between autonomy and communications often leads to sub-optimal compromises, resulting in reducing the already scarce communication bandwidth 
when autonomy is prioritised (e.g., repeat of redundant information), or to unnecessary mission interruptions to improve communications (e.g., rendezvous points, or surfacing).

Better and more integrated interactions between the autonomy and the communication stack would alleviate some of these problems. For example, in scenarios where the network stack provides communications as a service, through a well defined interface, this could be considered at the autonomy level as one of the available capabilities of the robot. The autonomy should not need the implementation details of the network stack, it should only need to use a series of generic commands to send and receive messages. Note that this is different to seeing communication as a potential bottleneck or constraint as was discussed before. The networks stack can also provide some additional services, such as localisation or channel modelling-again through a common interface. The stack can then be adjusted without the need for the autonomy to be changed. At the same time, the autonomy layer might also consider requests from the communication stack and use those, together with the other objectives, to define subsequent mission steps. In the case where the autonomy layer also provides its capabilities through services, requests from the communication stack (e.g., the routing layer that wants to move to a specific geographic location) can be seamlessly fused and weighted together with the rest of the objectives. The envisioned interplay between autonomy and communications is reported in Figure 7, with both autonomy and communications that make their functionalities available through dedicated services and a set of well defined interfaces. Passing information between the two systems is simplified and each system can request any of the available services and use the results to update internal data structures and objectives. In this architecture, each component has a minimum amount of data needed to function; however, it is clear to see the benefit of exposing other (optional) information for the system to make use of. For example: the physical layer is in the best position to infer metrics on the channel state, and the network layer may be able to maintain a topography of the locations of devices in the fleet. Utilising a well defined interface to share this information with the rest of the system, including the autonomy, would enable better modularity and increase its flexibility.

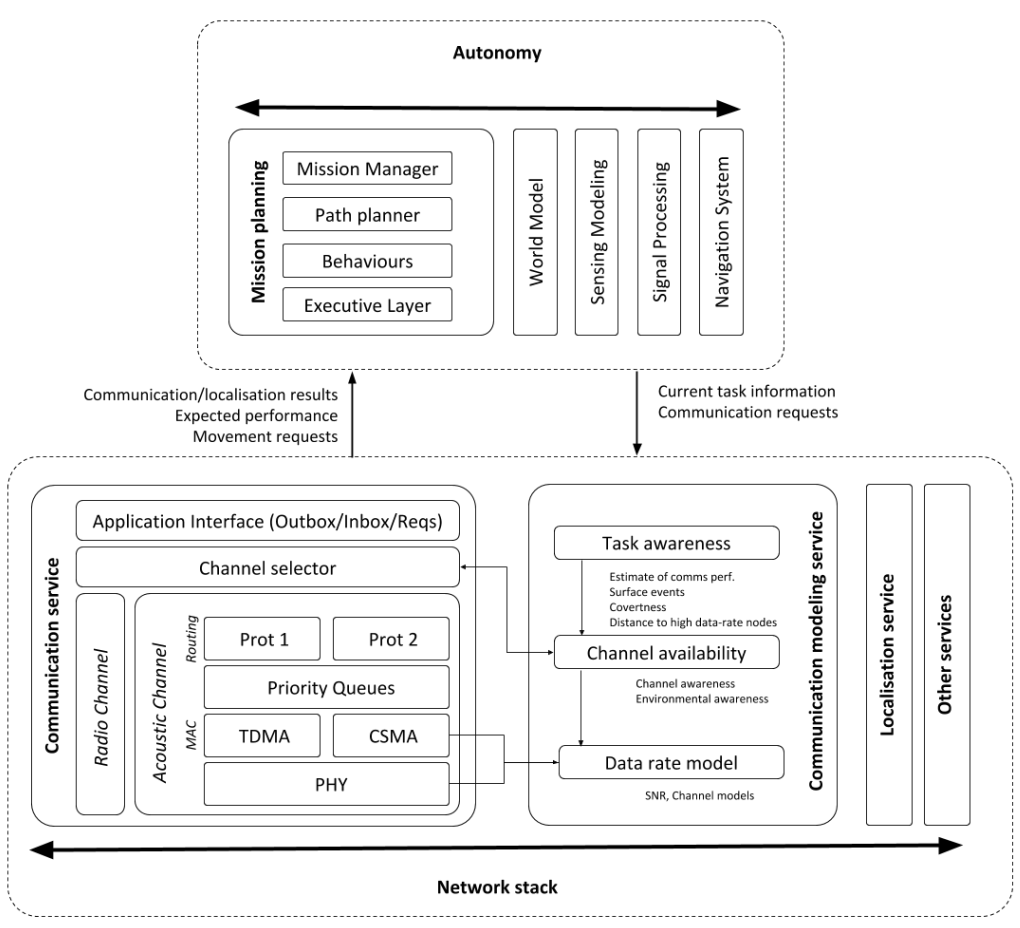

Figure 7. Interplay between autonomy and communications. Both systems make services available. Autonomy can call communication services (e.g., to transmit and receive), and the communication can also access autonomy services (e.g., the routing layer that wants the node to move to a specific geographic location). 


\section{A Successful Case Study: The Low-Cost AUV Technology (LCAT) Project}

The rise of a new generation of small, flexible and affordable AUVs, together with the development of miniaturised and low-cost sensors has given a strong push to the deployment of underwater networks. Having a strong interaction between the autonomy and the communication systems will allow the exploitation of the available data to be maximised, while overcoming the limitations imposed by the hardware. This is especially true for less expensive vehicles that cannot rely on high-power high-precision sensors.

This was, for example, the idea behind the development of the self-organising AUV network deployed by the Low-Cost AUV Technology (LCAT) project [48]. The project aimed at improving the functionalities of an existing underwater platform, the ecoSUB, enhancing its capabilities through network-based services. More specifically, due to their payload limitations, the LCAT vehicles cannot rely on external and possibly expensive sensors/infrastructure to support their mission, but rather they create an intelligent network that organises information and schedules AUV planning to maximise the in-mission performance. To support this, the AUVs rely on network services such as the communications/localisation service described in [6]. The rest of the section describes the deployed system and how the autonomy and communication layers were integrated together.

The ecoSUBs are small $(<1 \mathrm{~m},<12 \mathrm{~kg})$, long range $(+150 \mathrm{~km})$, deep rated $(2500 \mathrm{~m})$ AUVs that provide an unprecedented access to autonomous systems for use in science, commercial and defence applications through availability of low-cost systems and reduced infrastructure for launch, recovery and operation. The architecture of the on-board autonomy system is defined according to the front-seat/back-seat paradigm [49], and it is implemented using the functionalities offered by the open source Robot Operating System (ROS) middle-ware [50]. ROS is 'a collection of tools, libraries and conventions that aim to simplify the task of creating complex and robust robot behaviours across a wide variety of robotic platforms' [50]. It is very popular for terrestrial robotics platforms, especially in academia, and is gaining a lot of traction in the underwater robotics space. The central theme of ROS is of distributed and modular software components (called nodes) being developed separately, then working together using well defined messages (topics) to interact with each other. This design philosophy, as well as the already widespread usage, makes it an ideal platform to develop and share the previously proposed architecture.

The front-seat represents the vital part of the system and provides the vehicle with core components, such as mission planning, control, navigation, health and safety checks. On the other hand, the back-seat allows the system to be extended with additional, custom algorithms purposely designed to fit specific mission requirements, without affecting the safety of the vehicle. The intrinsic modularity of this framework facilitates the decomposition of the whole system into sub-modules, emphasising more the definition of interactions and interfaces between the different components rather than the distinctive features of the single entity.

Building on this insight, a back-seat driver enabling networked acoustic communication and enhanced navigation capabilities was designed to flank the existing front-seat of the ecoSUBs. The architecture of the LCAT system shown in Figure 8 has been adapted from the concepts discussed in Section 4 and envisioned in Figure 7 of a service-oriented modular architecture to make the communication and the autonomy layers interact.

The communication system provides the vehicle with a communication service and a localisation service. The autonomy system makes available a goto-waypoint and a navigation service that makes it possible for the vehicle to move to a specific geographical point.

The communication service includes:

- $\quad$ physical layer, represented by the nano-modems [51];

- data link layer, granting access to the acoustic channel by means of a configurable medium access control (MAC) policy. The user can select the MAC policy among the available ones, namely TDMA and Carrier-Sense Multiple Access (CSMA);

- network layer, providing basic addressing to each node; 
- application layer, interfaced with the network stack through a well-defined interface composed of an outbox and an inbox containing outgoing and incoming messages, respectively.

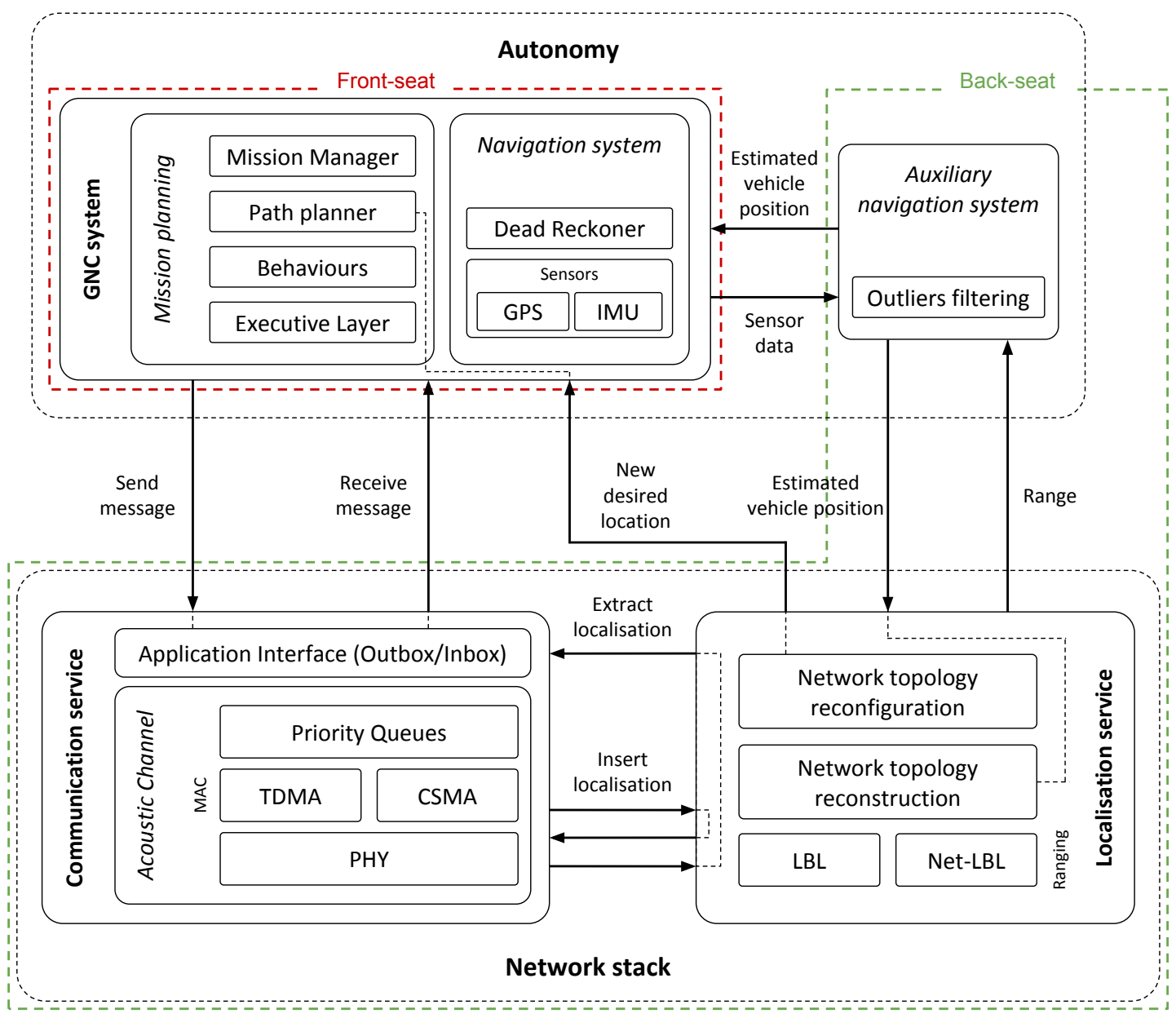

Figure 8. A customisation of the architecture proposed in Figure 7 for the Low-Cost AUV Technology (LCAT) project. The communication module provides the autonomy system with access to the acoustic channel, as well as with range measurements through the localisation service. The network stack can issue new waypoint requests to the path planner of the autonomy system (which includes a goto-waypoint service) in order to change the location of the asset.

The localisation service exploits inter-vehicle communications to evaluate measurements of the distance between the vehicle and another network node in an opportunistic manner, i.e., with a minimum impact on the normal functioning and without altering the main purpose of the network, as described in [6]. This ranging algorithm is based on an asynchronous message exchange (transaction) between two nodes. When the vehicle that started the transaction receives the answer back from the remote node, the range between them can be calculated on the basis of the Two-Way Travel Time (TWTT) and the sound speed, which, for the purpose of this experiment, was only measured once and assumed constant in the entire area. The overhead that has to be included in the messages to perform the range computation is appended to the next message scheduled for transmission whenever the available space allows it, removing the need for dedicated localisation packets. Range measurements, as well as the position of the corresponding node they refer to, are communicated to the autonomy system via a predefined interface as soon as they are available. Additionally, the localisation service embeds the capability of reconstructing the network topology, obtained as a by-product of the ranging algorithm. Note that while this feature is currently used in the system for monitoring purposes only, it can potentially be exploited by the communication system, e.g., to choose the best routing paths 
given the performance of the communication, or to determine the preferred location for the node that optimises one or more parameters. The service oriented architecture of the autonomy system would in fact make it easier to listen to requests coming from anywhere including the routing layer.

On the autonomy side, the auxiliary navigation system collects both proprioceptive (vehicle's speed and heading) and exteroceptive (ranges) measurements and fuses them together to obtain a refined navigation solution for the vehicle. The refined position estimate is then fed (a) to the primary navigation system of the ecoSUB's front-seat, and (b) back to the communication layer to inform the network reconstruction module of the current vehicle location.

The goto-waypoint service can be accessed by the localisation system to move the surface vehicles towards a target waypoint. This is used to maintain the desired network geometry and to keep the underwater vehicle in communication and localisation contact with the surface assets. While, for simplicity, this was statically defined during this experiment and based on a user-defined acceptance radius, the architectural elements were already in place to demonstrate the proposed concepts, and the requests could have been managed dynamically.

Figure 9 shows a deployment of the system during one of the experimental campaigns of the LCAT project conducted in Loch Ness, UK, in January 2019, where up to five vehicles were simultaneously deployed. A moderate water current (not measured and empirically assessed using the movement of the surface vehicles) from north-west to south-east was present throughout the experiment. In this preliminary deployment of the system, the network was constituted of three mobile nodes (Figure 9b), all fitted with the LCAT architecture shown in Figure 8. Two of them, with ID 12 (cyan) and 15 (yellow), were stationed on the surface drifting freely within a circular area with $70 \mathrm{~m}$ radius (black-shaded circles). Having access to the GPS signal, they were acting as reference beacons to aid the navigation of the third vehicle, with ID 157 (red). Vehicle 157 executed an underwater mission at $5 \mathrm{~m}$ depth consisting in two repetitions of the squared-shaped box (black line). While navigating underwater, the autonomy system of vehicle 157 was using the network provided localisation service to get range measurements with respect to the surface vehicles. The red line in Figure $9 \mathrm{~b}$ shows the real-time estimation of the vehicle position using the acoustic measurement. The error between the estimated position and the GPS at the final surfacing point was about $7 \mathrm{~m}$; running in post-processing the same mission without using acoustic ranges, the estimation error results of about $87 \mathrm{~m}$, an order of magnitude higher.

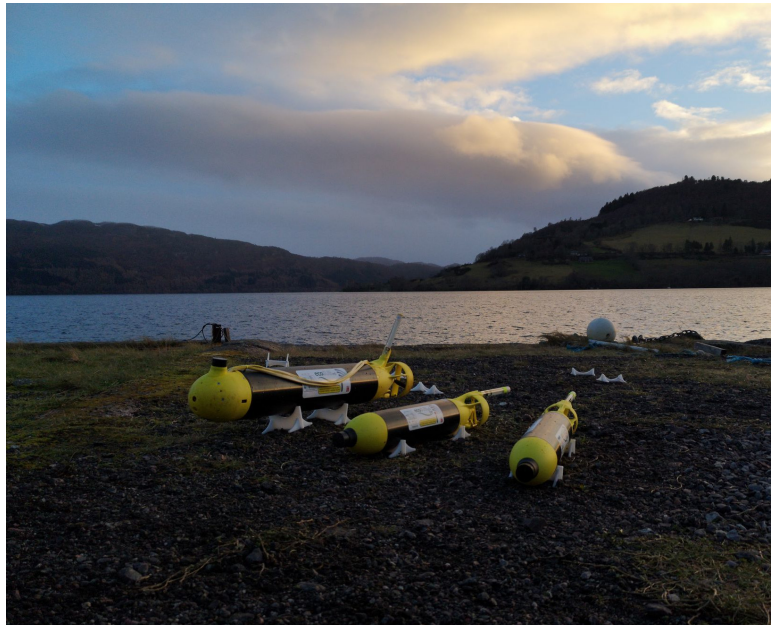

(a) ecoSUBs vehicles

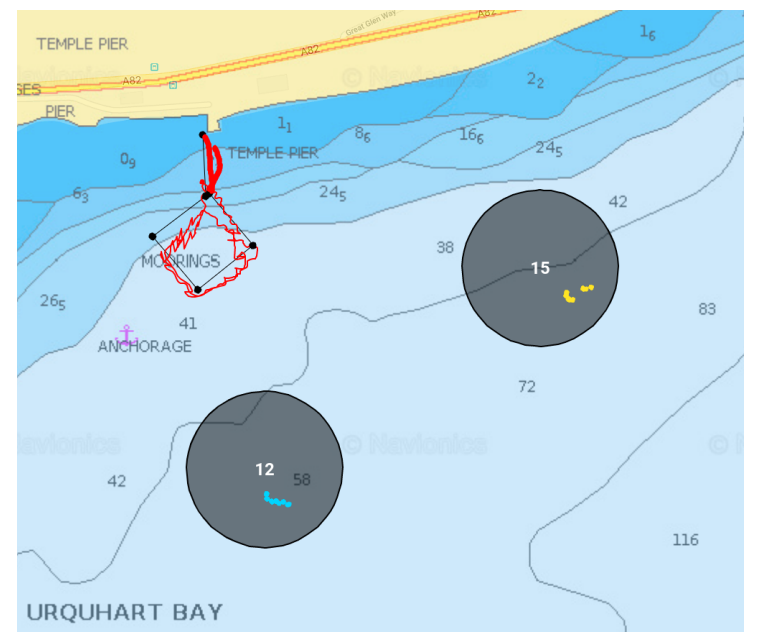

(b) Real-time localisation results

Figure 9. Experimental deployment of the system envisioned in the LCAT project in Loch Ness, UK, on 29 January 2019.

The availability of communication-enabled services at the autonomy level, allowed the AUVs to augment their navigation performance and, vice versa, at the communication/localisation level to keep 
the desired network geometry even in presence of water currents moving the surface assets away from their initial position. More broadly, this makes it possible to extend the range of use of the vehicles into areas which might preclude AUV technology (e.g., highly dynamic environments characterised by variable and/or strong currents, or deep waters where Doppler velocity log bottom-lock is difficult to obtain, etc.)

\section{Discussions}

Addressing the proposed use cases is not an easy feat, given the complexity of the missions, and of the environments. To tackle some of the limitations of the existing technology, this work proposes a tighter link between autonomy and communications. This makes it possible to simplify integration of different modules and to enhance the overall situational awareness of each robot/node, to include communications as well as sensing. In this regard, only a subset of the envisioned services have been implemented: communications, localisation and goto-waypoint, the latter being statically defined during the reported experiment and based on a user-defined acceptance radius. The architectural elements were, however, already in place and the waypoints requests could have been dynamically managed. For example, the proposed system would make it possible for the network stack to dynamically issue new waypoint requests to follow the underwater asset, or to change the geometry of the network to optimise its localisation. Implementing these services require the deployment of new autonomy modules that implement the necessary optimisation (see for example [19] and references therein). Importantly, however, the data and the infrastructure needed to make these more complex decisions at the autonomy level is already there, and a shorter implementation time can be expected for these new features.

The proposed approach represents a key enabler to ensure interoperability between the agents involved in the cooperative missions as envisioned in the presented use cases. Central to the deployment of interoperable systems, however, is the ability to develop and achieve standardisation across the whole autonomy and communications protocol stacks. This aspect has not been fully investigated in this paper, and more broadly is still relatively unexplored within the underwater community.

For example, from an acoustic communications perspective, each acoustic modem typically runs a manufacturers proprietary communications protocol. The only option for running a large scale network is to outfit all of the nodes with compatible modems. This rapidly inflates the cost of making changes to the modems on a vehicle-since changing manufacturer will require a complete refitting of the entire fleet to maintain full interoperability. Recent developments on open underwater communications standards have made significant headway in this area, for example the JANUS protocol [52] (developed by the CMRE) is an open source underwater communication protocol, which is already available from some manufacturers. Whilst there is some adoption, it is far from being a ubiquitous standard. This lack of flexibility extends into the design of the networking stack. There are ongoing efforts to develop new techniques for underwater communications, some of which have been highlighted in this paper and others are shown in the literature. These describe novel adaptations to elements of the protocol stack, such as adaptive physical layers or smart networking protocols. To actually implement these requires integration with existing systems, which is often a slow process, requiring applications to be customised to take advantage of the new communications solution. Much like any peripheral device, each modem has a unique interface. Whilst similarities do exist between similar product lines, each modem does require an integration activity to enable access from the platforms code base. This makes integration tasks much slower, as the communications stack must be re-written to cope with the specifics of the modem being used. Many systems do not utilise a separate communications stack; instead they make the communications behaviour a core part of the application. This can make development easier, but means that a change in modem hardware requires a rewrite of the application. All of this contributes to raising the bar of entry needed to develop applications to run on underwater systems. A designer (or their team) must have knowledge of not only the desired system, but also of 
the entire communications stack. A deeper discussion on interoperability issues is out of the scope of this work, and the reader is referred to [53] for more details on these aspects.

In the proposed service-oriented architecture, the interface between each layer and module is realised through messages, with the common standard being enforced through message type definitions. The same system can be used for optional data and a program implementing a particular module is able to push any ancillary information it generates to a message bus open to the rest of the system. All of these messages would be accessible by any arbitrary program that would be free to read data from any part of the communications and autonomy stacks. This includes both the optional extra data as well as the core communications data. This is not without its problems. The most pressing of which is the situation when a program in the system relies on an optional message type that is not actually implemented. This is similar to an issue of duplicate data types; for example, two different developers could have two different implementations of depth data. Whilst not ideal, a possible solution is the creation of well publicised community guidelines and libraries. These would give a shared implementation of the most common data types, as well as provide suggestions of how to handle optional data types.

A more complex problem is that of data fusion. How a system combines data from sensors producing similar data types is an unsolved problem, and one that will have an impact on this architecture. By listening for a particular message type, a node will need to deal with data being sent from multiple other nodes, and somehow decide how best to utilise this information.

\section{Conclusions}

Throughout this paper, the authors have explored the constraints and implications of UWA networks and how as we move forward into an era of autonomy; these networks can be adapted by AUV manoeuvrability to ensure that robust and reliable communications can be achieved.

Two uses cases are presented to motivate the work and to highlight the advantages that a tighter link between autonomy and communications can bring to the outcome of the mission. One specific example that builds on the work done under the Innovate UK LCAT project is provided that shows that a strong interaction between the autonomy and the communication system allows to overcome some of the limitation imposed by the vehicle hardware. The communication service was leveraged by the localisation to provide navigational updates to the vehicle. In turn, the goto-waypoint service made available by the autonomy was exploited to keep the network geometry even in presence of water current that would have moved the surface assets away from the rest of the network. For experimental simplicity, the goto-waypoint was used through statically defined service calls but the architecture was able to support dynamic requests. Future experimentation will investigate the addition of more services that would enable more complex decision making.

From a communication perspective, a promising first step forward is the promulgation of STANAG 4748, commonly known as JANUS [54] and adoption of this across nations. However, JANUS is simply the first step on a long road to achieving secure interoperability in underwater autonomous networks and presents one method for initial contact. To have secure interoperable and adaptable autonomous networks, new protocols must be developed at all levels to be able to support future requirements concerning key aspects such as data rate, robustness, agility and security. The importance of these new approaches is highlighted by recent international efforts, such as the NATO Science and Technology Office (STO) Research Task Group (RTG) Information and Systems Technology (IST) Panel 174-Secure Underwater Communications for Heterogeneous Networks. This effort, led by Dstl, is explicitly tackling these problems, to jointly develop enhancements and new protocols, and to support the development of enhanced underwater communications networks.

Author Contributions: Use cases, A.H. and S.H.; channel sensing and network adaptation, N.M. and P.M.; data mule, A.H.; tasking the network, D.F., A.M. and J.S.; discussions, A.H., S.H., A.M. and D.F. All authors have read and agreed to the published version of the manuscript. 
Funding: The work of Nils Morozs and Paul Mitchell was supported by the UK Engineering and Physical Sciences Research Council (EPSRC) through the USMART (EP/P017975/1) and Full-Duplex (EP/R003297/1) projects.

Acknowledgments: D. Fenucci, A. Munafò and J. Sitbon would like to thank Jeff Neasham and the Newcastle University LCAT team for their support with the acoustic nanomodems used during the Loch Ness experiment, Terry Sloane, Iain Vincent and Alex Downer from Planet Ocean Ltd./ecoSUB Robotics Ltd. and Alexander B. Phillips from the National Oceanography Centre for their help with the ecoSUB vehicles, and everyone that participated in the Innovate UK funded project 'LCAT': Low Cost AUV Technology: development of smart networks and AI Based Navigation for dynamic underwater environments, project reference 104058.

Content includes material subject to (c) Crown copyright (2020), Dstl. This material is licensed under the terms of the Open Government Licence v3.0 except where otherwise stated. To view this licence, visit http://www.nationalarchives.gov.uk/doc/open-government-licence/version/3.

Conflicts of Interest: The authors declare no conflict of interest.

\section{Abbreviations}

The following abbreviations are used in this manuscript:

$\begin{array}{ll}\text { AUV } & \text { Unmanned Underwater Vehicle } \\ \text { UWA } & \text { Underwater Acoustic Communications } \\ \text { UAN } & \text { Underwater Acoustic Networks } \\ \text { UASN } & \text { Underwater Acoustic Sensor Networks } \\ \text { RTS } & \text { Request-to-Send } \\ \text { CTS } & \text { Clear-to-Send } \\ \text { C2 } & \text { Command and Control } \\ \text { MCM } & \text { Mine Counter Measures } \\ \text { ASW } & \text { Anti-Submarine Warfare } \\ \text { MAC } & \text { Medium Access Control } \\ \text { DSSS } & \text { Direct Sequence Spread Spectrum } \\ \text { FH-FSK } & \text { Frequency-Hopped Frequency Shift Keying } \\ \text { SSP } & \text { Sound Speed Profile } \\ \text { SNR } & \text { Signal-to-Noise Ratio } \\ \text { H\&D } & \text { Harvest and Deliver } \\ \text { IMU } & \text { Inertial Measurement Unit } \\ \text { TDMA } & \text { Time Division Multiple Access } \\ \text { CDMA } & \text { Code Division Multiple Access } \\ \text { LBL } & \text { Long Base Line } \\ \text { ROS } & \text { Robot Operating System [50] } \\ \text { CMRE } & \text { Centre for Maritime Research and Experimentation } \\ \text { STO } & \text { Science and Technology Organisation } \\ \text { IST } & \text { Information and Systems Technology } \\ \text { RTG } & \text { Research Task Group }\end{array}$

\section{References}

1. $\quad$ Lloyd Butler VK5BR. Underwater radio communication. Amat. Radio 1987.

2. Melodia, T.; Kulhandjian, H.; Kuo, L.C.; Demirors, E.; Basagni, S.; Conti, M.; Giordano, S.; Stojmenovic, I. Mobile Ad Hoc Networking: Cutting Edge Directions; John Wiley and Sons: Hoboken, NJ, USA, 2013; Advances in underwater acoustic networking, pp. 804-852.

3. Song, A.; Stojanovic, M.; Chitre, M. Editorial underwater acoustic communications: Where we stand and what is next? IEEE J. Ocean. Eng. 2019, 44, 1-6. [CrossRef]

4. Caiti, A.; Crisostomi, E.; Munafò, A. Sensor Systems and Software; Springer: Berlin, Heidelberg, 2010; Physical Characterization of Acoustic Communication Channel Properties in Underwater Mobile Sensor Networks, pp. 111-126.

5. $\quad$ Ferri, G.; Munafò, A.; Tesei, A.; Braca, P.; Meyer, F.; Pelekanakis, K.; Petroccia, R.; Alves, J.; Strode, C.; LePage, K. Cooperative robotic networks for underwater surveillance: An overview. IET Radar Sonar Navig. 2017, 11, 1740-1761. [CrossRef]

6. Munafò, A.; Ferri, G. An acoustic network navigation system. J. Field Rob. 2017, 34, 1332-1351. [CrossRef] 
7. Caiti, A.; Fabbri, T.; Fenucci, D.; Munafò, A. Potential games and AUVs cooperation: First results from the THESAURUS project. In Proceedings of the 2013 MTS/IEEE OCEANS - Bergen, Bergen, Norway, 10-14 June 2013; pp. 1-6.

8. abiani, F.; Fenucci, D.; Caiti, A. A distributed passivity approach to AUV teams control in cooperating potential games. Ocean Eng. 2018, 157, 152-163. [CrossRef]

9. Yordanova, V.; Griffiths, H.; Hailes, S. Rendezvous planning for multiple autonomous underwater vehicles using a Markov decision process. IET Radar Sonar Navig. 2017, 11, 1762-1769. [CrossRef]

10. Yao, Z.; Gupta, K. Distributed roadmaps for robot navigation in sensor networks. In Proceedings of the 2010 IEEE International Conference on Robotics and Automation, Anchorage, AK, USA, 4-8 May 2010; pp. 3078-3083. [CrossRef]

11. Ltd, S. Neptune Website. Available online: https://www.seebyte.com/products/neptune/ (accessed on 1 June 2020).

12. Heshmati-Alamdari, S.; Bechlioulis, C.P.; Karras, G.C.; Kyriakopoulos, K.J. Cooperative impedance control for multiple underwater vehicle manipulator systems under lean communication. IEEE J. Oceanic Eng. 2020. [CrossRef]

13. Simetti, E.; Casalino, G. Manipulation and transportation with cooperative underwater vehicle manipulator systems. IEEE J. Ocean. Eng. 2017, 42, 782-799. [CrossRef]

14. Basagni, S.; Bölöni, L.; Gjanci, P.; Petrioli, C.; Phillips, C.A.; Turgut, D. Maximizing the value of sensed information in underwater wireless sensor networks via an autonomous underwater vehicle. In Proceedings of the 33rd Annual IEEE International Conference on Computer Communications, Toronto, Canada, 27 April-2 May 2014; pp. 988-996. [CrossRef]

15. Petroccia, R.; Zappa, G.; Furfaro, T.; Alves, J.; D’Amaro, L. Development of a software-defined and cognitive communications architecture at CMRE. In Proceedings of the OCEANS 2018 MTS/IEEE Charleston, Charleston, SC, USA, 22-25 October 2018; pp. 1-10. [CrossRef]

16. Schneider, T.; Schmidt, H. Unified command and control for heterogeneous marine sensing networks. J. Field Rob. 2010, 27, 876-889. [CrossRef]

17. Rahmati, M.; Arjula, A.; Pompili, D. Compressed underwater acoustic communications for dynamic interaction with underwater vehicles. In Proceedings of the International Conference on Underwater Networks \& Systems, Atlanta, GA, USA, 23-25 October 2019; pp. 1-5 doi:10.1145/3366486.3366488. [CrossRef]

18. Chen, B.; Pompili, D. The Art of Wireless Sensor Networks: Volume 2: Advanced Topics and Applications; Springer: Heidelberg, Germany, 2014; A Communication Framework for Networked Autonomous Underwater Vehicles, pp. 485-525._13. [CrossRef]

19. Munafò, A.; Sliwka, J.; Alves, J. Dynamic placement of a constellation of surface buoys for enhanced underwater positioning. In Proceedings of the OCEANS'15 MTS/IEEE Genova, Genova, Italy, 18-21 May 2015; pp. 1-6. [CrossRef]

20. Willners, J.S.; Toohey, L.; Petillot, Y. Sampling-based path planning for cooperative autonomous maritime vehicles to reduce uncertainty in range-only localization. IEEE Robot. Autom. Lett. 2019, 4, 3987-3994. [CrossRef]

21. Ridolfi, A.; Spaccini, D.; Fanelli, F.; Franchi, M.; Monni, N.; Picari, L.; Petrioli, C.; Allotta, B. An autonomous underwater vehicle and SUNSET to bridge underwater networks composed of multi-vendor modems. Annu. Rev. Control 2018, 46, 295-303. [CrossRef]

22. Schneider, T.; Schmidt, H. Model-based adaptive behavior framework for optimal acoustic communication and sensing by marine robots. IEEE J. Ocean. Eng. 2013, 38, 522-533. [CrossRef]

23. Chitre, M.; Shahabudeen, S.; Stojanovic, M. Underwater acoustic communications and networking: Recent advances and future challenges. Mar. Technol. Soc. J. 2008, 42, 103-116.

24. Amar, A.; Avrashi, G.; Stojanovic, M. Low complexity residual doppler shift estimation for underwater acoustic multicarrier communication. IEEE Trans. Signal Process. 2017, 65, 2063-2076. [CrossRef]

25. Qu, F.; Wang, Z.; Yang, L.; Wu, Z. A journey toward modeling and resolving doppler in underwater acoustic communications. IEEE Commun. Mag. 2016, 54, 49-55. [CrossRef]

26. Wilson, W.D. Speed of sound in sea water as a function of temperature, pressure, and salinity. J. Acoust. Soc. Am. 1960, 32, 641-644. [CrossRef] 
27. Dushaw, B. Worldwide Sound Speed, Temperature, Salinity, and Buoyancy from the NOAA World Ocean Atlas. Available online: Http://staff.washington.edu/dushaw/WOA/ (accessed on 1 June 2020).

28. Morozs, N.; Gorma, W.; Henson, B.; Shen, L.; Mitchell, P.D.; Zakharov, Y. Channel modeling for underwater acoustic network simulation. IEEE Access 2020, 8, 136151-136175.

29. Porter, M. Beam tracing for two- and three-dimensional problems in ocean acoustics. J. Acoust. Soc. Am. 2019, 146, 2016-2029. [CrossRef]

30. Zakharov, Y.; Henson, B.; Diamant, R.; Fei, Y.; Mitchell, P.; Morozs, N.; Shen, L.; Tozer, T. Data packet structure and modem design for dynamic underwater acoustic channels. IEEE J. Ocean. Eng. 2019, 44, 837-849. [CrossRef]

31. Stojanovic, M. On the relationship between capacity and distance in an underwater acoustic communication channel. SIGMOBILE Mob. Comput. Commun. Rev. 2007, 11, 34-43. [CrossRef]

32. Jensen, F.; Kuperman, W.; Porter, M.; Schmidt, H. Computational Ocean Acoustics; Springer: New York, USA, 2011.

33. Caiti, A.; Munafò, A.; Petroccia, R. Encyclopedia of Robotics; Springer: Heidelberg, Germany, 2020; Underwater Communication, pp. 1-10._14-1. [CrossRef]

34. Caiti, A.; Grythe, K.; Hovem, J.M.; Jesus, S.M.; Lie, A.; Munafò, A.; Reinen, T.A.; Silva, A.; Zabel, F. Linking acoustic communications and network performance: Integration and experimentation of an underwater acoustic network. IEEE J. Ocean. Eng. 2013, 38, 758-771. [CrossRef]

35. Heidemann, J.; Stojanovic, M.; Zorzi, M. Underwater sensor networks: Applications, advances and challenges. Philos. Trans. R. Soc. A 2012, 370, 158-175. [CrossRef]

36. Molins, M.; Stojanovic, M. Slotted FAMA: A MAC protocol for underwater acoustic networks. In Proceedings of the IEEE Oceans 2006-Asia Pacific, Singapore, 16-19 May 2006.

37. Noh, Y.; Lee, U.; Han, S.; Wang, P.; Torres, D.; Kim, J.; Gerla, M. DOTS: A propagation delay-aware opportunistic MAC protocol for mobile underwater networks. IEEE Trans. Mob. Comput. 2014, 13, 766-782. [CrossRef]

38. Anjangi, P.; Chitre, M. Propagation-delay-aware unslotted schedules with variable packet duration for underwater acoustic networks. IEEE J. Ocean. Eng. 2017, 42, 977-993. [CrossRef]

39. Diamant, R.; Lampe, L. Spatial reuse time-division multiple access for broadcast ad hoc underwater acoustic communication networks. IEEE J. Ocean. Eng. 2011, 36, 172-185. [CrossRef]

40. Lmai, S.; Chitre, M.; Laot, C.; Houcke, S. Throughput-efficient super-TDMA MAC transmission schedules in ad hoc linear underwater acoustic networks. IEEE J. Ocean. Eng. 2017, 42, 156-174. [CrossRef]

41. Vermeij, A.; Munafó, A. Clock synchronisation in underwater acoustic networks. In Proceedings of the 2014 Underwater Communications and Networking (UComms 2014), Sestri Levante, Italy, 3-5 September 2014 ; pp. 1-5.

42. Sozer, E.M.; Stojanovic, M.; Proakis, J.G. Underwater acoustic networks. IEEE J. Ocean. Eng. 2000, 25, 72-83. [CrossRef]

43. Pompili, D.; Melodia, T.; Akyildiz, I.F. A CDMA-based medium access control for underwater acoustic sensor networks. IEEE Trans. Wirel. Commun. 2009, 8, 1899-1909. [CrossRef]

44. Freitag, L.; Stojanovic, M.; Singh, S.; Johnson, M. Analysis of channel effects on direct-sequence and frequency-hopped spread-spectrum acoustic communication. IEEE J. Ocean. Eng. 2001, 26, 586-593. [CrossRef]

45. Freitag, L.; Johnson, M.; Grund, M.; Singh, S.; Preisig, J. Integrated acoustic communication and navigation for multiple UUVs. In Proceedings of the MTS/IEEE OCEANS'01, Honolulu, HI, USA, 5-8 November 2001; pp. 2065-2070.

46. Lal, C.; Petroccia, R.; Pelekanakis, K.; Conti, M.; Alves, J. Toward the development of secure underwater acoustic networks. IEEE J. Ocean. Eng. 2017, 42, 1075-1087. [CrossRef]

47. Li, J.; Yang, B.; Ye, D.; Wang, L.; Fu, K.; Piao, J.; Wang, Y. A real-time, full-duplex system for underwater wireless optical communication: Hardware structure and optical link model. IEEE Access 2020, 8, 109372-109387. [CrossRef]

48. Fenucci, D.; Munafò, A.; Phillips, A.B.; Neasham, J.; Gold, N.; Sitbon, J.; Vincent, I.; Sloane, T. Development of smart networks for navigation in dynamic underwater environments. In Proceedings of the 2018 IEEE/OES Autonomous Underwater Vehicle Workshop (AUV), Porto, Portugal, 6-9 November 2018; pp. 1-6. [CrossRef] 
49. Eickstedt, D.P.; Sideleau, S.R. The backseat control architecture for autonomous robotic vehicles: A case study with the Iver2 AUV. Mar. Technol. Soc. J. 2010, 44, 42-54. [CrossRef]

50. Robotics, O. ROS.Org I Powering the World's Robots. Available online: https://www.ros.org/ (accessed on 1 March 2020).

51. Phillips, A.B.; Gold, N.; Linton, N.; Harris, C.A.; Richards, E.; Templeton, R.; Thuné, S.; Sitbon, J.; Muller, M.; Vincent, I.; et al. Agile design of low-cost autonomous underwater vehicles. In Proceedings of the OCEANS 2017, Aberdeen, UK, 19-22 June 2017; pp. 1-7.

52. CMRE. Janus Wiki. Available online: http://www.januswiki.com/tiki-index.php (accessed on 1 March 2020).

53. Costanzi, R.; Fenucci, D.; Manzari, V.; Micheli, M.; Morlando, L.; Terracciano, D.; Caiti, A.; Stifani, M.; Tesei, A. Interoperability among unmanned maritime vehicles: Review and first in-field experimentation. Front. Robot. AI 2020, 7, 91. [CrossRef]

54. Potter, J.; Alves, J.; Green, D.; Zappa, G.; McCoy, K.; Nissen, I. The JANUS underwater communications standard. In Proceedings of the 2014 Underwater Communications and Networking (UComms), Sestri Levante, Italy, 3-5 September 2014; pp. 1-4. [CrossRef]

(C) 2020 by the authors. Licensee MDPI, Basel, Switzerland. This article is an open access article distributed under the terms and conditions of the Creative Commons Attribution (CC BY) license (http://creativecommons.org/licenses/by/4.0/). 